\title{
Frequency-dependent AVO attribute: theory and example
}

Xiaoyang $\mathrm{Wu}^{1 *}{ }^{\text {* }}$ Mark Chapman ${ }^{1,2}$ and Xiang-Yang $\mathrm{Li}^{1}$

${ }^{1}$ Edinburgh Anisotropy Project, British Geological Survey, Murchison House, West Mains Road, Edinburgh EH9 3LA, UK.

${ }^{2}$ School of Geosciences, University of Edinburgh, The King's Buildings, West Mains Road, Edinburgh EH9 3JW, UK.

*Corresponding author,E-mail:xywu@bgs.ac.uk

\begin{abstract}
Fluid-saturated rocks generally have seismic velocities that depend upon frequency. Exploring this property may help us discriminate different fluids from seismic data. In this paper, we introduce a scheme to calculate a frequency-dependent AVO attribute in order to estimate seismic dispersion from pre-stack data, and apply it to North Sea data. The scheme essentially combines the two-term approximation of Smith and Gidlow (1987) with the method of spectral decomposition based on the Wigner-Ville distribution, which is used to achieve high resolution. The result suggests the potential of this method for detection of seismic dispersion due to fluid saturation.
\end{abstract}




\section{Introduction}

Fluid substitution using Gassmann's theory is the core of most seismic methods for fluid detection. However, the elastic behaviour of fluid-saturated rocks can be frequency-dependent. A variety of rock physical models (Mavko and Nur, 1975; Dvorkin and Nur, 1993; Chapman et al., 2002) have been developed to describe dispersion and attenuation of seismic waves in fluid-saturated media. Squirt flow is considered to be the dominant mechanism for attenuation and dispersion of laboratory data. Batzle et al. (2006) observed strong seismic velocity dispersion from seismic to ultrasonic frequencies in the laboratory and considered the dispersion to be controlled by fluid mobility, which is defined as the ratio of rock permeability to fluid viscosity. Analysis of seismic data suggests that hydrocarbon accumulations are often associated with abnormally high values of attenuation, which is generally not accounted for by Gassmann's theory and amplitude-versus-offset (AVO) analysis.

Application of spectral decomposition techniques allows frequency-dependent behaviour to be detected in seismic data, and reflections from hydrocarbon-saturated zones can be anomalous in this regard (Castagna et al., 2003). A variety of spectral decomposition techniques, such as the short time Fourier transform (STFT), continuous wavelet transform (CWT), matching pursuit decomposition (MPD), and Wigner-Ville distribution (WVD) methods, have been studied and used for different applications, including layer thickness determination (Partyka et al., 1999), stratigraphic visualization (Marfurt and Kirlin, 2001), as well as direct hydrocarbon detection. STFT, CWT and MPD methodologies can be considered as atomic decomposition, which decompose non-stationary signals as the linear combination of time-frequency atoms. Castagna et al. (2006) compared a series of atomic decomposition 
methods and concluded that MPD has the best combination of temporal and spectral resolution. An alternative class of spectral decomposition is the energy distribution, which distributes the energy of a signal with a function dependent on two variables: time and frequency. The representative WVD is well recognized as an effective method for time-frequency analysis of non-stationary signals. The WVD of the real signal $x(t)$ can be defined as $W V D(t, f)=\int_{-\infty}^{\infty} X(t+\tau / 2) \bar{X}(t-\tau / 2) e^{-j 2 \pi f \tau} d \tau$,

where $\tau$ is the time delay variable and $X(t)$ is the analytical signal associated with $x(t)$. Being quadratic in nature, WVD introduces cross terms for a multicomponent signal. However, cross-term interference can be smoothed away by using kernel functions. Wu and Liu (2009) compared WVD with STFT and CWT and found that the smoothed pseudo WVD (SPWVD) using two Gaussian functions to suppress cross-term interference provided higher temporal and frequency resolution than STFT and CWT.

Chapman et al. (2005) performed a theoretical study of reflections from layers which exhibit fluid-related dispersion and attenuation, and showed that in such cases the AVO response was frequency-dependent. Wilson et al. (2009) extended this analysis, introducing a frequency-dependent AVO (FAVO) inversion aimed at allowing a direct measure of dispersion to be derived from pre-stack data. In this paper, we combine this FAVO inversion scheme with high-resolution SPWVD, and present a new attribute to estimate seismic dispersion from pre-stack data. Field data application from the North Sea also hints at the potential of this method to be used in detection of seismic dispersion. 


\section{Theory}

The two-term AVO approximation of Smith and Gidlow (1987) can be written as $R(\theta) \approx A(\theta) \frac{\Delta V_{p}}{V_{p}}+B(\theta) \frac{\Delta V_{s}}{V_{s}}$,

where $\theta$ is the angle of incidence, and $A(\theta)$ and $B(\theta)$ can be derived in terms of the known velocity model. Following the theory of Wilson et al. (2009), in the frequency-dependent attribute scheme the reflection coefficient $R(\theta)$ and the (fractional) contrasts in P-wave and S-wave impedances, $\Delta V_{p} / V_{p}$ and $\Delta V_{s} / V_{s}$, are considered to vary with frequency due to dispersion in the material. Then Equation 2 can be written as:

$$
R(\theta, f) \approx A(\theta) \frac{\Delta V_{p}}{V_{p}}(f)+B(\theta) \frac{\Delta V_{s}}{V_{s}}(f) .
$$

Expanding Equation 3 as a first-order Taylor series around a reference frequency $f_{0}$,

$$
R(\theta, f) \approx A(\theta) \frac{\Delta V_{p}}{V_{p}}\left(f_{0}\right)+\left(f-f_{0}\right) A(\theta) I_{a}+B(\theta) \frac{\Delta V_{s}}{V_{s}}\left(f_{0}\right)+\left(f-f_{0}\right) B(\theta) I_{b},
$$

where $I_{a}$ and $I_{b}$ are the derivatives of the velocity contrasts with respect to frequency evaluated at $f_{0}$ :

$$
I_{a}=\frac{d}{d f}\left(\frac{\Delta V_{p}}{V_{p}}\right) \text { and } I_{b}=\frac{d}{d f}\left(\frac{\Delta V_{s}}{V_{s}}\right)
$$

Given that seismic amplitudes can be associated with the reflection coefficients through convolution with a seismic wavelet in the AVO analysis, according to Equation 3, we derive $\Delta V_{p} / V_{p}$ and $\Delta V_{s} / V_{s}$ at the reference frequency $f_{0}$ by replacing $R\left(\theta, f_{0}\right)$ with a suitably balanced spectral amplitude at $f_{0} . \quad I_{a}$ and $I_{b}$ can be derived from Equation 4 by using least-squares method for the spectral amplitudes at a series of frequencies. When $R(\theta, f)$ is replaced by the spectral amplitude at different frequencies, $I_{a}$ and $I_{b}$ describe the derivative of 
the seismic velocity contrasts with frequency, related to P-wave dispersion and S-wave dispersion. Since the spectral amplitude is positive, we cannot handle phase reversals, and only the absolute values of $I_{a}$ and $I_{b}$ are meaningful. Considering that shear modulus is usually independent of the saturating fluid, we focus on the $I_{a}$ attribute to estimate the magnitude of P-wave dispersion, which is our new FAVO attribute. The SPWVD method is used for spectral decomposition.

\section{Synthetic example}

We study a two-layer synthetic case that considers the effect of varying dispersive properties in the materials, based on the models created by Chapman et al. (2006). The parameters of the model are listed in Table 1. The model contains a time-scale parameter $\tau$ which is proportional to fluid mobility and which controls the frequency range over which dispersion occurs. The upper elastic shale layer has P-wave velocity of $2743 \mathrm{~m} \mathrm{~s}^{-1}$, S-wave velocity of $1394 \mathrm{~ms}^{-1}$, and a density of $2.06 \mathrm{~g} \mathrm{~cm}^{-3}$. The lower sandstone layer is considered to have P-wave velocity of $2835 \mathrm{~m} \mathrm{~s}^{-1}$, S-wave velocity of $1472 \mathrm{~m} \mathrm{~s}^{-1}$, and a density of $2.08 \mathrm{~g} \mathrm{~cm}^{-3}$ under water saturation.

Eleven receivers were synthesized with a trace spacing of $100 \mathrm{~m}$ and $40 \mathrm{~Hz}$ Ricker wavelet as the explosive source. Figure 1 displays the four synthetic gathers generated by ANISEIS software package with a Fortran program to create the frequency-dependent layer. Two dispersive cases under water and gas saturation respectively are considered with the same $\tau$ value of $5 \times 10^{-3} \mathrm{~s}$. The interface of low frequency case $\left(\tau=1 \times 10^{-6} \mathrm{~s}\right)$ and the high frequency case ( $\tau=100 \mathrm{~s})$ can be considered to be elastic. From Figure 1a, we can see the 
typical Class I AVO feature that the amplitudes decrease with offset under water saturation. Class I AVO changes to Class III AVO when water is substituted with gas. Another feature when gas-saturated is that the amplitudes decrease with increasing $\tau$ value.

Figure 2 displays the $I_{a}$ attribute estimated for each of the four models by application of our FAVO method. The method correctly determines the magnitude of dispersion is the highest in case $b$.

\section{Data example}

The workflow for the scheme to calculate the FAVO attribute $I_{a}$ is shown in Figure 3. For the post-stack data, we perform SPWVD to obtain the spectral amplitude at a series of frequencies, and then perform spectral balancing to remove the overprint of the wavelet. We can then analyse the change of spectral amplitude with frequency. For pre-stack data, we perform the same workflow as that on post-stack data. The known stacking velocities are used to estimate the layer velocities using Dix's formula, so that the angles of incidence can be estimated by ray tracing.

In order to select the optimal frequencies and reference frequency for spectral decomposition and the FAVO, we analysed the energy distribution of two arbitrary pre-stack and stacked traces with the Fast Fourier transform (Figure 4). The dominant frequency is around $15 \mathrm{~Hz}$ with bandwidth of $0-40 \mathrm{~Hz}$ for pre-stack traces. There is low-frequency interference at the near surface, which is suppressed after stacking. Therefore, we defined the reference frequency as $15 \mathrm{~Hz}$ and selected the set of frequencies 10, 15, 20, 30, and $40 \mathrm{~Hz}$ for the FAVO attribute. 
A cross-line stacked section from the North Sea is shown in Figure 5(a). The area of interest is around $2.0 \mathrm{~s}$ at a depth of about $2 \mathrm{~km}$. There are three potentially hydrocarbon-related accumulations at three bright spots, numbered 1, 2 and 3, where the pre-stack CMP gathers exhibit Class III and Class IV AVO anomalies. We performed SPWVD on the stacked section to obtain iso-frequency sections at 10, 15, 20, 30, and $40 \mathrm{~Hz}$. Continuous reflections above $1.0 \mathrm{~s}$ were used for spectral balancing with $15 \mathrm{~Hz}$ as the reference frequency. The resultant balanced sections at 10, 15, 20, 30, and $40 \mathrm{~Hz}$ are plotted in Figure 5b-f using a consistent amplitude scale that allows us to directly compare the different spectral amplitudes. The spectral amplitudes at bright spot 1 have an increasing trend with frequency, whilst spectral amplitudes at bright spots 2 and 3 are stable at 10, 15, and 20 $\mathrm{Hz}$ and then decrease at 30 and $40 \mathrm{~Hz}$. In addition, bright spot 3 can be divided into two parts, the spectral amplitude of the first part, 3(i) at CMPs 950-1079, is weaker than that of the second part, 3(ii) at CMPs 1080-1190. Table 2 summarizes the interpretation of the CMP gathers along the horizon. Bright spots 1 and 2 have small amplitudes at the near offsets which increase with offset. They can be interpreted as Class III reflections. Bright spot 3(i) has large negative amplitude at the near-offsets and the amplitude decreases with offset, i.e., the gradient is positive, so it is interpreted as a Class IV response. Bright spot 3(ii) has negative amplitude at near-offsets that increases with offset, and it is interpreted as a Class III response. These different AVO anomalies may be related to velocity contrasts due to fluid saturation variation in the reservoir.

The pre-stack CMP gathers of the cross-line were extracted from the 3D seismic data for the FAVO analysis. In order to make the attribute estimation accurate, true seismic amplitude 
variation with offset must be contained in each CMP gather, and the influence of NMO stretch must be suppressed. We used the first 45 traces in each CMP gather for the FAVO. The trace spacing is $75 \mathrm{~m}$ and the maximum offset is $3686 \mathrm{~m}$. The angle of incidence on CMP gathers were calculated from the known velocity model, and are displayed for CMP 700 as an example in Figure 6. The angles of incidence around $2.0 \mathrm{~s}$ are $0-16^{\circ}$, substantially less than $30^{\circ}$, which is the requirement for the two-term AVO approximation of Smith and Gidlow (1987). Nevertheless, the first 45 traces are able to delineate the changing seismic amplitude with offset.

We performed SPWVD on the pre-stack gathers to obtain iso-frequency sections at 10 , 15, 20, 30, and 40Hz. Figure 7 displays CMP gather 700 located at bright spot 2 with the $I_{a}$ attribute plotted at the right. At $2.0 \mathrm{~s}$, the amplitudes exhibit the typical features of a class III AVO response. Figure 8 displays our FAVO attribute $I_{a}$ for CMPs 202-1600. We can see many of the reflections have been suppressed. The zone of interest around $2.0 \mathrm{~s}$ shows a significant magnitude of the $I_{a}$ attribute.

\section{Conclusions}

Fluid-related dispersion and attenuation gives rise to frequency-dependent reflection coefficients, and in many cases can have a strong effect on reflection data. This paper combines a high-resolution spectral decomposition technique with a frequency-dependent AVO attribute scheme to demonstrate the possibility of inferring dispersion properties directly from pre-stack data and linking this to fluid saturation. We illustrate the method through analysis of synthetic data. The real seismic example from the North Sea indicates the 
potential of this method for the detection of seismic dispersion resulting from fluid saturation. The use of appropriate rock physics models to directly estimate fluid properties from such analysis is a topic of current research.

\section{Acknowledgements}

We are grateful to David Taylor for providing the code for reflectivity modelling with frequency-dependent properties (ANISEIS). This work was supported by the sponsors of the Edinburgh Anisotropy Project.

\section{References}

Batzle, M.L., Han, D.-H. and Hofmann, R. [2006] Fluid mobility and frequency-dependent seismic velocity, direct measurements. Geophysics, 71, N1-N9.

Castagna, J.P. Sun, S. and Siegfried, R.W. [2003] Instantaneous spectral analysis: detection of low-frequency shadows associated with hydrocarbons, The Leading Edge, 22, 120-127. Castagna, J.P. and Sun, S. [2006] Comparison of spectral decomposition methods. First Break, 24(3), 75-79.

Chapman, M., Liu, E. and Li, X.-Y. [2006] The influence of fluid-sensitive dispersion and attenuation on AVO analysis. Geophysical Journal International, 167, 89-105.

Chapman, M., Zatsepin, S.V. and Crampin, S. [2002] Derivation of a microstructural poroelastic model. Geophysical Journal International, 151, 427-451.

Dvorkin, J. and Nur, A. [1993] Dynamic poroelasticity: a unified model with the squirt and the Biot mechanisms. Geophysics, 58, 524-533. 
Marfurt, K.J. and Kirlin, R.L. [2001] Narrow-band spectral analysis and thin-bed tuning. Geophysics, 66, 1274-1283.

Mavko, G. and Nur, A. [1975] Melt squirt in the asthenosphere. Journal of Geophysical Research, 80, 1444-1448.

Partyka, G.A., Gridley, J.M. and Lopez, J. [1999] Interpretational applications of spectral decomposition in reservoir characterization. The Leading Edge, 18, 353-360.

Smith, G.C. and Gidlow, P.M. [1987] Weighted stacking for rock property estimation and detection of gas. Geophysical Prospecting, 35, 993-1014.

Wilson, A., Chapman, M. and Li, X.-Y. [2009] Frequency-dependent AVO inversion. $79^{\text {th }}$ SEG Meeting, Expanded Abstracts, 28, 341-345.

$\mathrm{Wu}, \mathrm{X}$. and Liu, T. [2009] Spectral decomposition of seismic data with reassigned smoothed pseudo Wigner-Ville distribution. Journal of Applied Geophysics, 68, 386-393.

Wu, X., Chapman, M., Wilson, A. and Li, X.-Y. [2010] Estimating seismic dispersion from pre-stack data using frequency-dependent AVO inversion. $80^{\text {th }}$ SEG Meeting, Expanded Abstracts, 29, 425-429.

Table 1 Material parameters for the two-layer model.

\begin{tabular}{|c|c|c|c|c|c|c|c|}
\hline Lithofacies & $\begin{array}{c}V_{p} \\
\left(\mathrm{~m} \mathrm{~s}^{-1}\right)\end{array}$ & $\begin{array}{c}V_{s} \\
\left(\mathrm{~m} \mathrm{~s}^{-1}\right)\end{array}$ & $\begin{array}{c}\rho_{\mathrm{W}} \\
\left(\mathrm{g} \mathrm{cm}^{-3}\right)\end{array}$ & $\begin{array}{c}\rho_{\mathrm{G}} \\
\left(\mathrm{g} \mathrm{cm}^{-3}\right)\end{array}$ & $\begin{array}{c}\text { Porosity } \\
(\%)\end{array}$ & $\begin{array}{c}\text { Crack } \\
\text { density }(\%)\end{array}$ & $\begin{array}{c}\text { Thickness } \\
(\mathrm{m})\end{array}$ \\
\hline Shales & 2743 & 1394 & 2.06 & - & - & - & 1000 \\
\hline Sandstone & 2835 & 1472 & 2.08 & 2.04 & 15 & 5 & half space \\
\hline
\end{tabular}


Table 2 Interpretation of the CMP gathers at the positions of three bright spots.

\begin{tabular}{|c|c|c|c|c|}
\hline Bright spot & CMP range & Amplitude & versus offset & Interpretation \\
\hline 1 & $350-430$ & negative & increasing & Class III \\
\hline 2 & $610-840$ & negative & increasing & Class III \\
\hline $3($ i $)$ & $950-1080$ & negative & decreasing & Class IV \\
\hline $3($ ii) & $1080-1190$ & negative & increasing & Class III \\
\hline
\end{tabular}
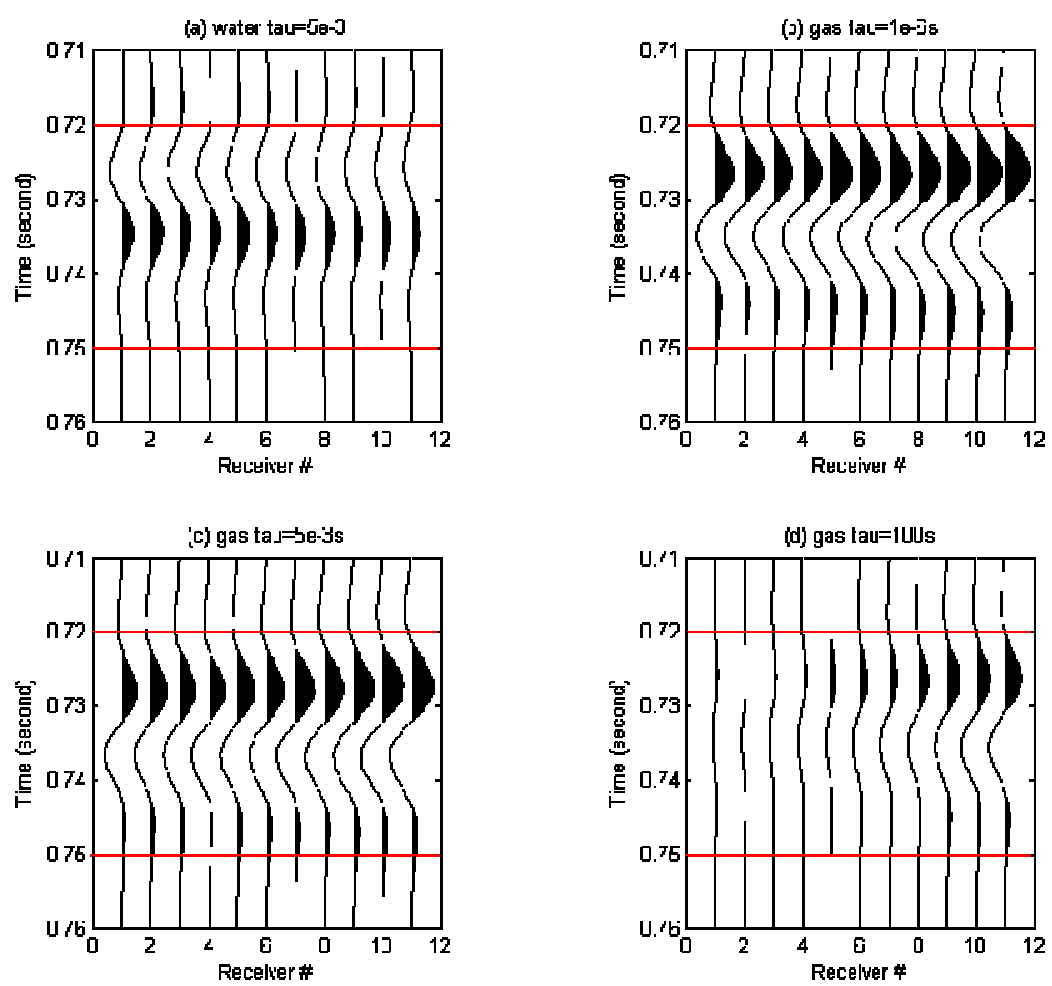

Figure 1 Four synthetic gathers under (a) water saturation $\left(\tau=5 \times 10^{-3} \mathrm{~s}\right)$, (b) gas saturation $\left(\tau=1 \times 10^{-6} \mathrm{~s}\right)$, (c) gas saturation $\left(\tau=5 \times 10^{-3} \mathrm{~s}\right)$ and $(\mathrm{d})$ gas saturation $(\tau=100 \mathrm{~s})$. 


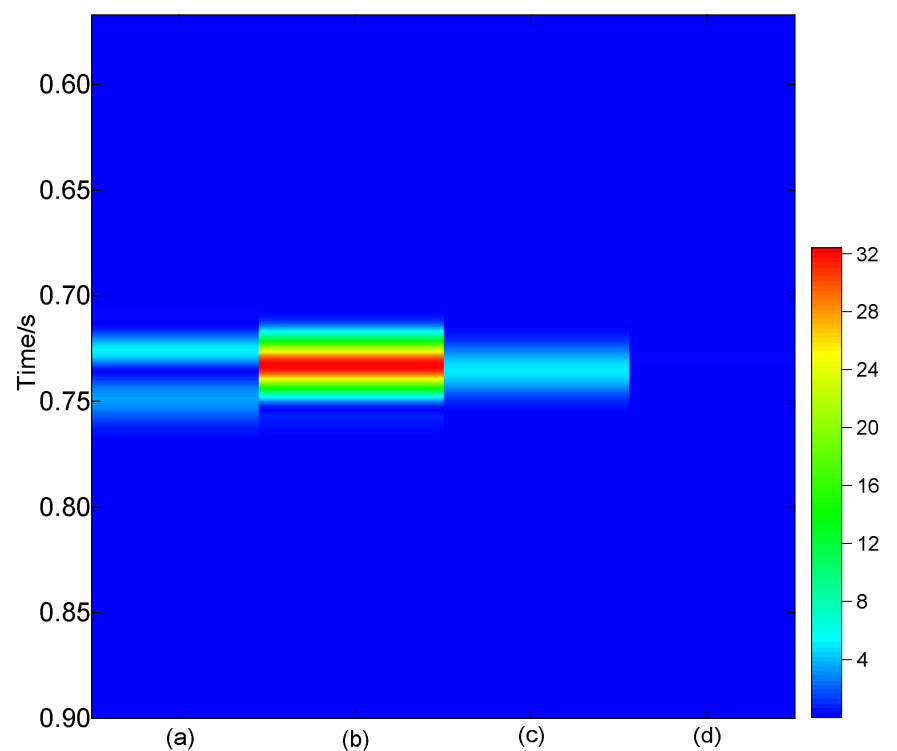

Figure 2 The $I_{a}$ attribute for the four synthetics. (a) gas saturation $\left(\tau=1 \times 10^{-6} \mathrm{~s}\right)$, (b) gas saturation $\left(\tau=5 \times 10^{-3} \mathrm{~s}\right)$, (c) water saturation $\left(\tau=5 \times 10^{-3} \mathrm{~s}\right)$ and (d) gas saturation $(\tau=100 \mathrm{~s})$.

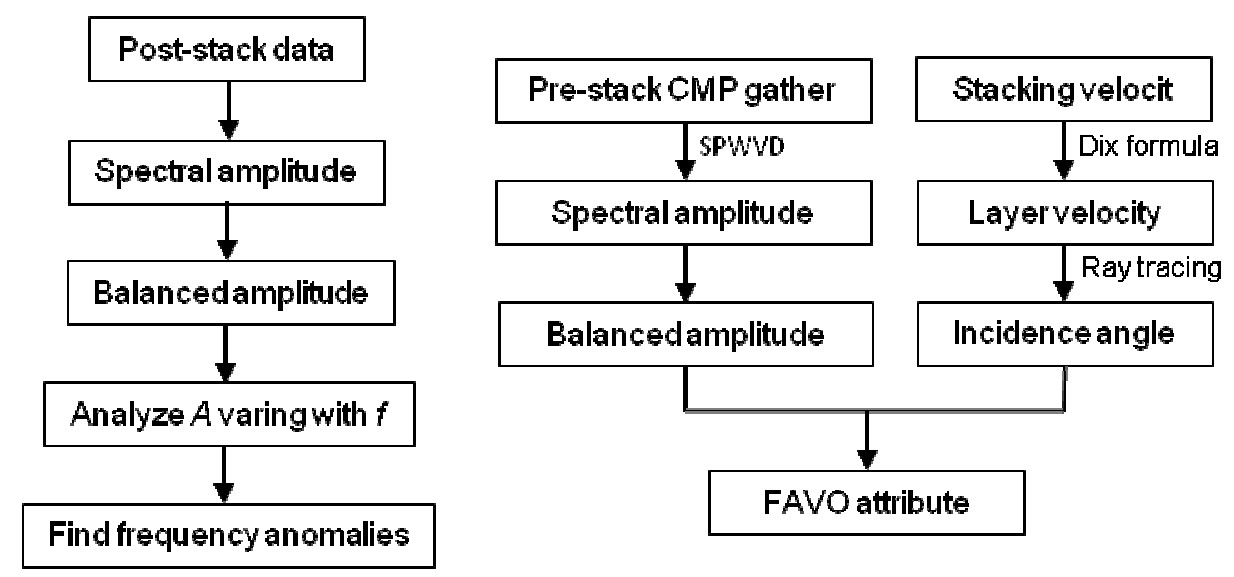

Figure 3 The workflow for FAVO attribute estimation scheme.
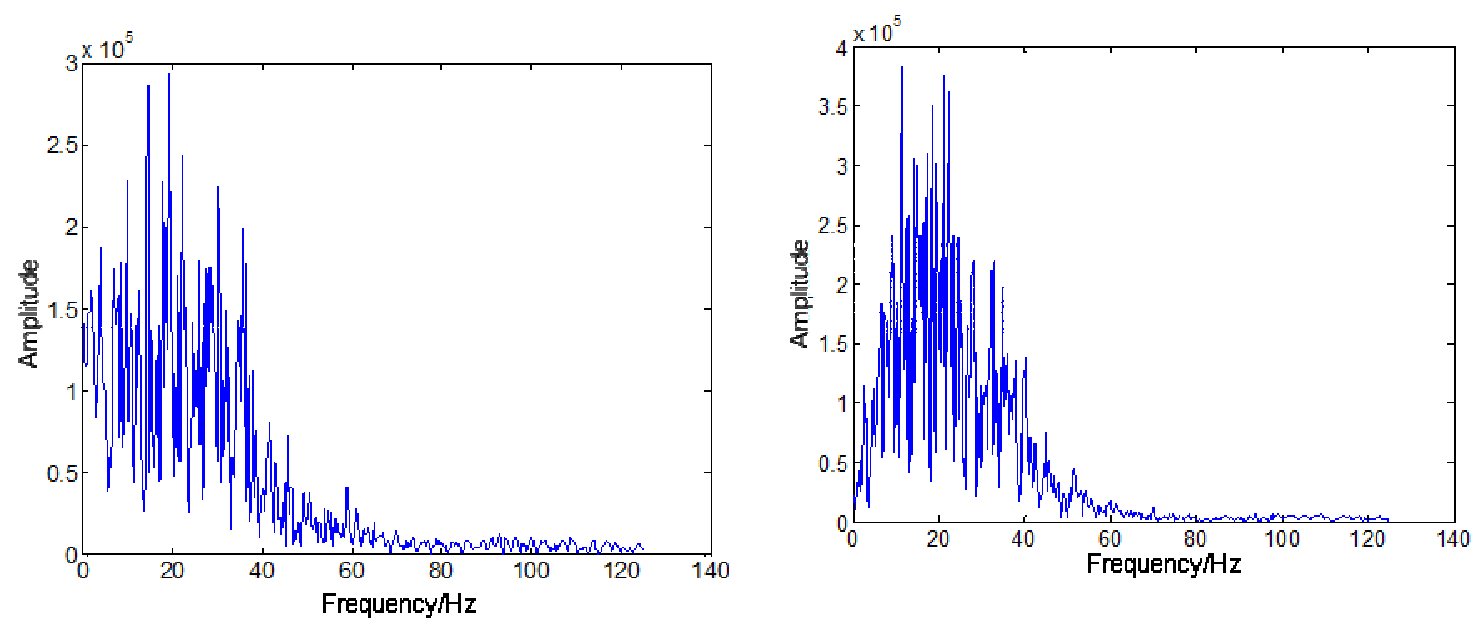
pre-stack trace

stacked trace

Figure 4 Spectral analysis of two arbitrary traces using FFT.

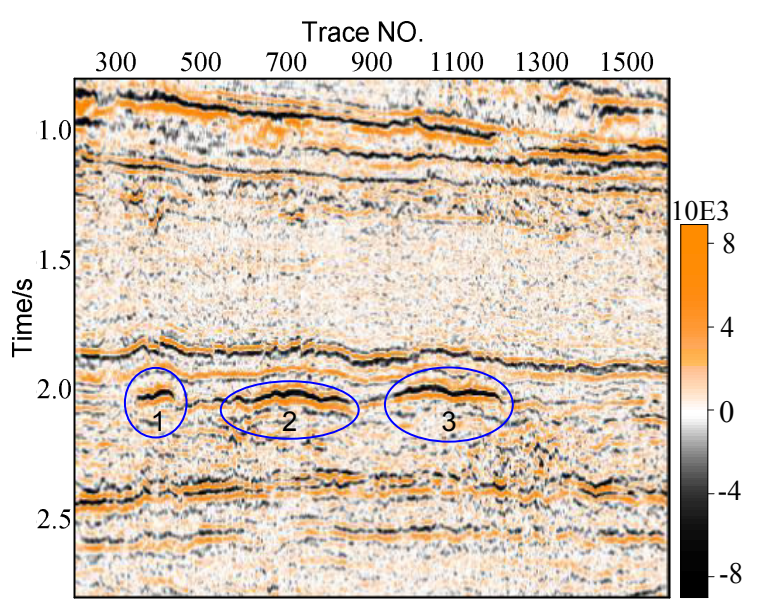

(a) Post-stack section of a cross-line Trace NO.

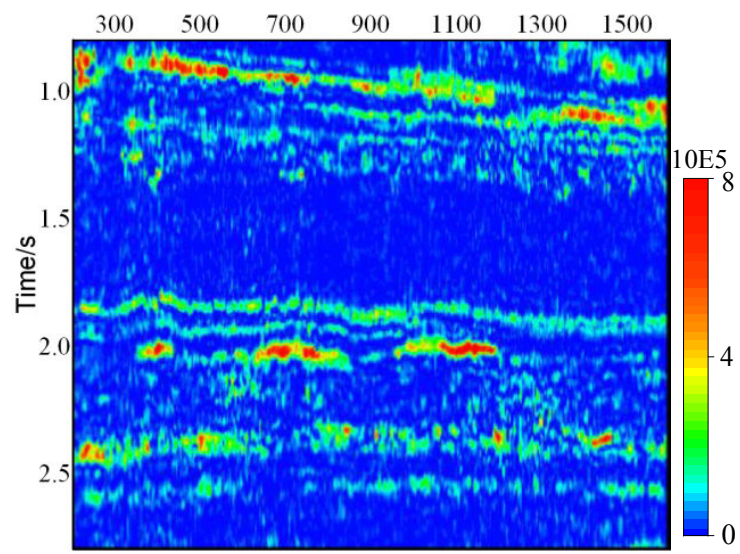

(c) $15 \mathrm{~Hz}$

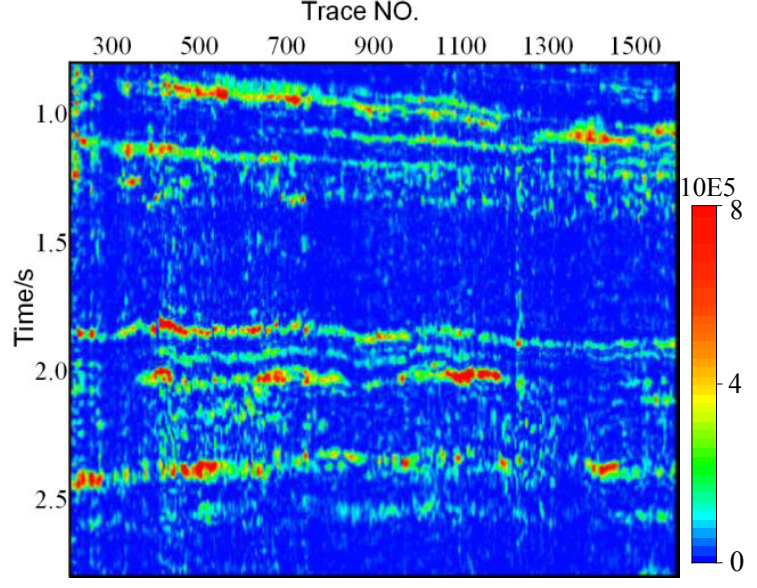

(e) $30 \mathrm{~Hz}$

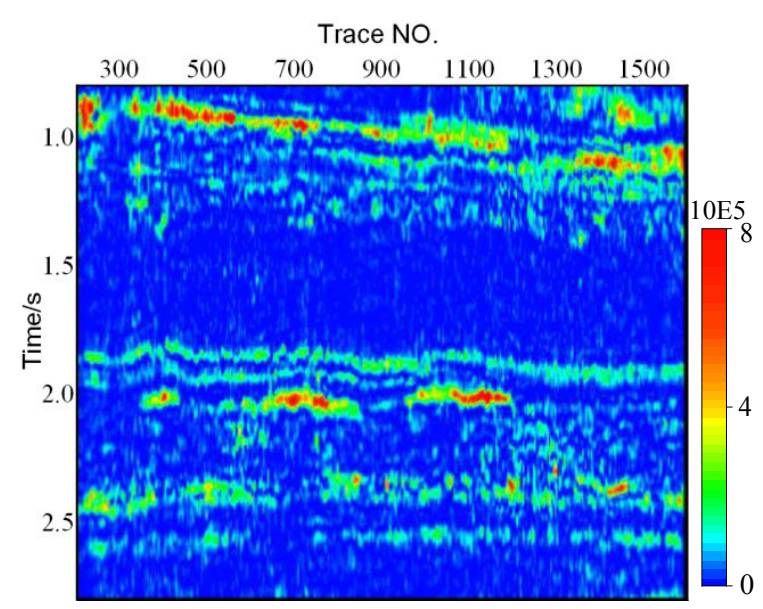

(b) $10 \mathrm{~Hz}$

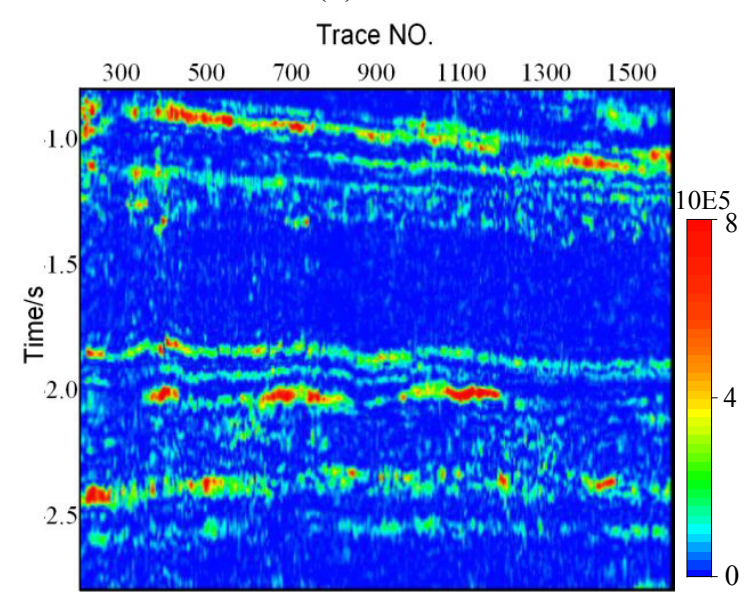

(d) $20 \mathrm{~Hz}$

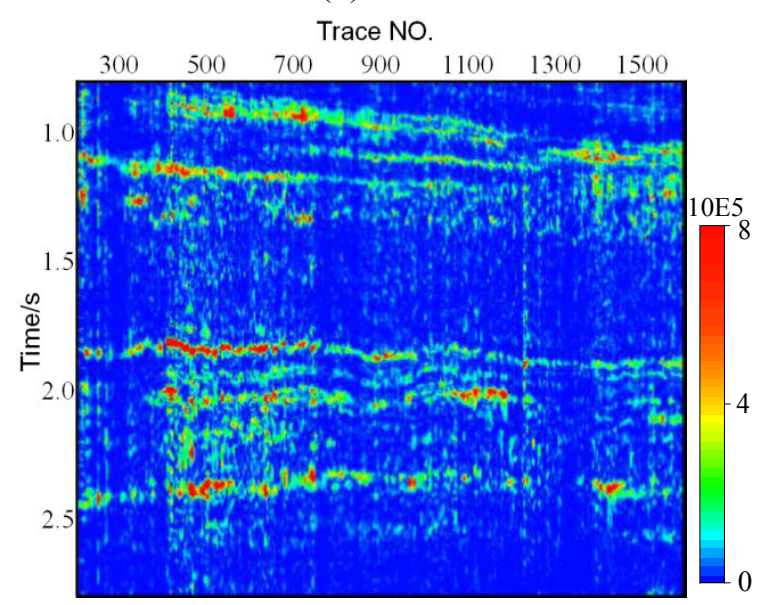

(f) $40 \mathrm{~Hz}$

Figure 5 Post-stack section of the cross-line and its iso-frequency sections. 


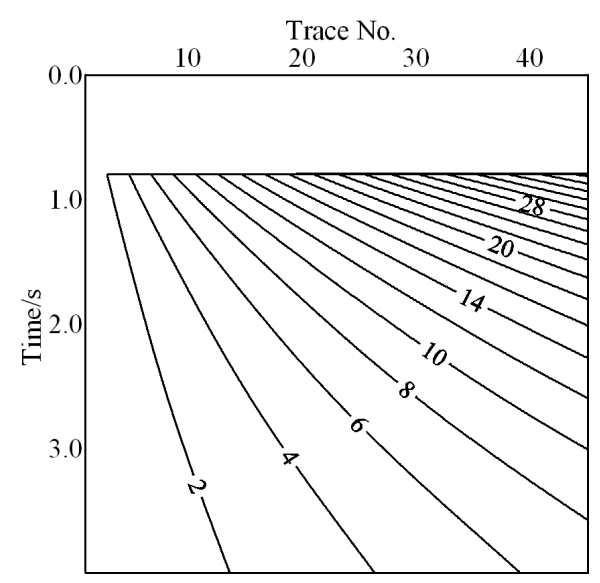

Figure 6 Incident angle in degrees for CMP gather 700 calculated from the known velocity model.

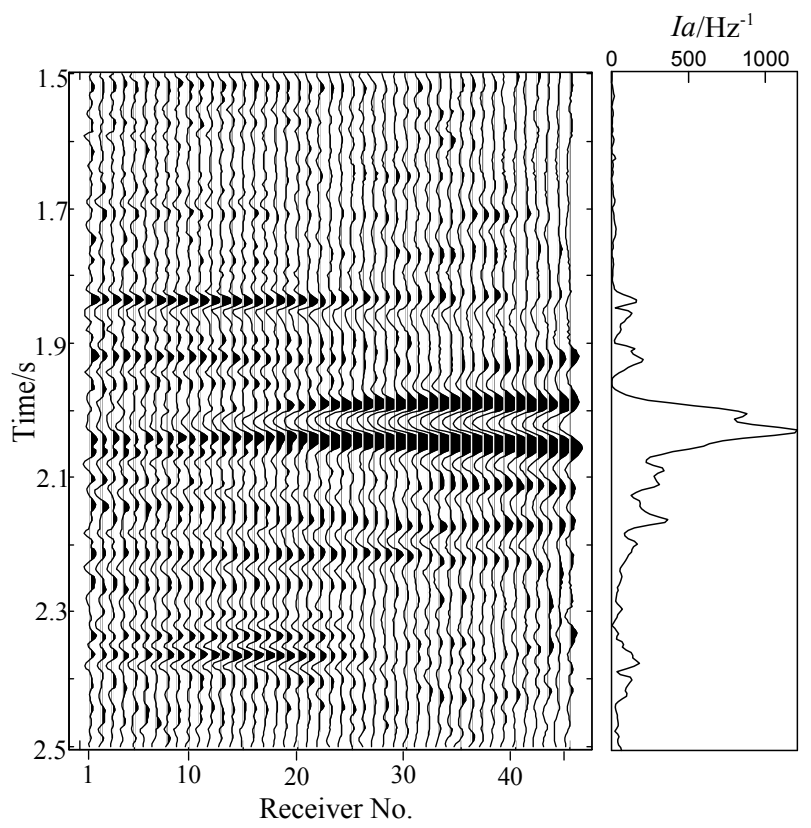

Figure 7 The $I_{a}$ attribute for No.700 CMP gather (from No.2 anomaly).

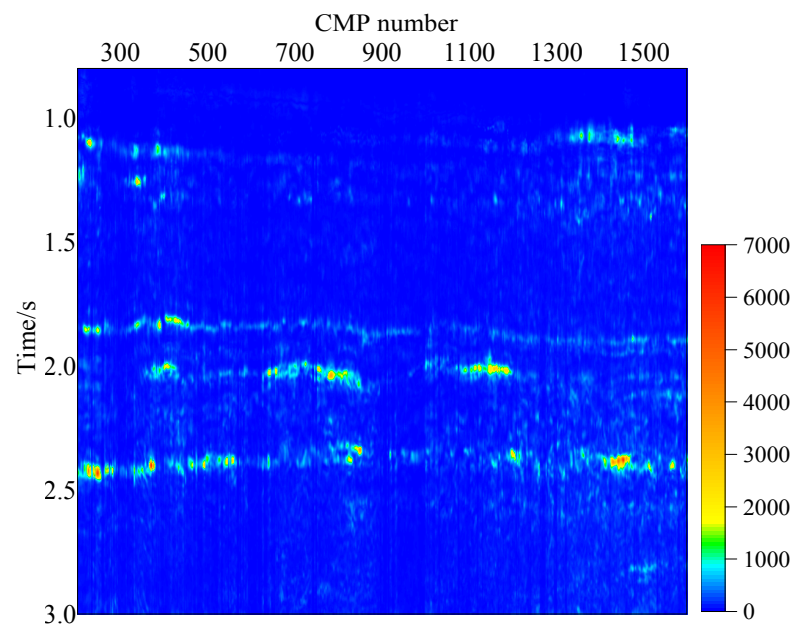

Figure 8 The $I_{a}$ attribute for the cross-line (unit: $\mathrm{Hz}^{-1}$ ). 